\section{Cytotoxic T Lymphocyte-associated Antigen 4 Ig-induced Asthma in the Treatment of Rheumatoid Arthritis}

\section{To the Editor}

The CD28-CD80/CD86 costimulatory pathway is essential in enhancing and optimizing $\mathrm{T}$ cell activation and cytokine production in rheumatoid arthritis (RA). Cytotoxic T lymphocyte-associated antigen 4 (CTLA4) is a homolog of CD28. It can competitively bind to CD80/CD86 and downregulate naive and memory $\mathrm{T}$ cell proliferation, cytokine production, and humoral immune response. Its human soluble fusion protein, CTLA4-Ig, was approved for the treatment of active RA by the US Food and Drug Administration in $2005^{1}$. Several animal studies suggest benefits of CTLA4-Ig in other immunologic diseases such as asthma. However, we describe here 2 cases of RA complicated with relapse of asthma while undergoing CTLA4-Ig therapy.

A 53-year-old Chinese woman had a 9-year history of refractory RA, and was treated with methotrexate (MTX) $7.5 \mathrm{mg}$ per week and prednisone $3 \mathrm{mg}$ daily. She had started CTLA4-Ig $10 \mathrm{mg} / \mathrm{kg}$ in combination with stable dosages of MTX and prednisone on August 20, 2009, because of her high RA activity. Four days later, she presented with progressive cough, tachypnea, and chest distress. She did not complain of fever, rash, or joint pain. Although she was diagnosed with asthma in 1989, she did not experience any episodes of asthma in the previous 20 years. Upon admission, the laboratory examination revealed an abnormal arterial blood gas with partial pressure of oxygen $53.3 \mathrm{~mm} \mathrm{Hg}$, partial pressure of carbon dioxide $33.9 \mathrm{~mm} \mathrm{Hg}$, and oxygen saturation $88.7 \%$ on room air. The chest radiograph was normal. No evidence of bacteria (including mycobacterium tuberculosis) or fungi was noted. Her chest examination revealed diffuse wheezing in all lung fields. Episode of asthma was diagnosed. Then CTLA4-Ig was stopped, and intravenous methylprednisolone $80 \mathrm{mg} / \mathrm{day}$ was initiated with concurrent bronchodilators. The symptoms of asthma disappeared in 5 days. Steroid was tapered gradually during followup. She has had no recurrence of asthma.

In another case, a 53-year-old Chinese woman was diagnosed with RA in 1989. She still had active arthritis after multiple therapies including infliximab infusion and MTX treatment. She had no other systemic complaints although she had had a few episodes of asthma during her childhood. She received 4 doses of CTLA4-Ig $10 \mathrm{mg} / \mathrm{kg}$ at Weeks $0,2,4$, and 8, combined with MTX $10 \mathrm{mg}$ weekly between July 30 and September 24, 2009. She had a good response to CTLA4-Ig, with $50 \%$ improvement according to the American Rheumatism Association criteria when receiving the fourth infusion. However, she developed dry cough by October 2009. Although orally treated with antibiotics and antitussive, her cough worsened, accompanied by wheezing and shortness of breath. Chest examination revealed diffuse wheezes in all lung fields. She had no fever and no arthritis flare. Complete blood count was normal. She was diagnosed with asthma at the emergency department of our hospital on October 20. She stopped CTLA4-Ig therapy and was treated with bronchodilators and oral prednisone $15 \mathrm{mg}$ twice per day. Her asthma was resolved by October 30, and she gradually tapered prednisone to $10 \mathrm{mg} /$ day.

In a published clinical trial of CTLA4-Ig (ASSURE), an increased risk of respiratory adverse effects, such as cough, rhonchi, exacerbation of chronic obstructive pulmonary disease (COPD), dyspnea, and nasal congestion, has been reported in patients with RA with underlying COPD ${ }^{2}$. Two cases of severe respiratory complications associated with CTLA4-Ig have been described in patients with RA who had been previously diagnosed with COPD and asthma, but the former case developed fungal pneu- monia and the latter, receiving 9-month CTLA4-Ig treatment, had begun MTX 1 month before asthma flared ${ }^{3}$. However, in our 2 cases, no infection was found. They had no other systemic complaints except active arthritis before CTLA4-Ig treatment. They had not developed asthma for at least 20 years until they received CTLA4-Ig infusion. The mechanism underlying these side effects is unknown. We are not certain that the modulation of costimulatory molecules is relevant to the recurrence of asthma.

Th2 cells play a central role in the initiation and progression of allergic asthma. One therapeutic strategy for asthma is the prevention of activation of allergen-specific Th2 cells by blocking their costimulatory signaling. CTLA4-Ig has been widely used to disrupt $\mathrm{T}$ cell costimulation in a number of experimental animal models of $\mathrm{T}$ cell-mediated inflammatory diseases, such as asthma ${ }^{4}$. Deurloo, et al investigated the role of CTLA4-Ig in murine models of established asthma that had been sensitized by ovalbu$\min ^{5}$. However, the results showed that CTLA4-Ig had no effect on airway inflammation in severe models, although it reversed asthma manifestations in mild ones. Lordan, et $a l^{6}$ revealed that the secretion of Th2 cytokines, such as interleukin 5, was largely independent of CD28-mediated costimulation in patients with moderately severe asthma. The findings suggest there may be other mechanisms of costimulation pathway or $\mathrm{T}$ cell activation for asthma. Further investigation should be done.

CTLA4-Ig should be used cautiously in patients with a history of asthma, because it may induce recurrence of asthma.

JUN BAO, MD, PhD; TING LI, MD; JING ZHAO, MD; CAI-YING ZHANG, MD, Department of Rheumatology and Immunology, Changzheng Hospital, Second Military Medical University, 415 Fengyang Road, Shanghai 200003, China. Address correspondence to Dr. J. Bao; E-mail: baojun@smmu.edu.cn

\section{REFERENCES}

1. Food and Drug Administration. Orencia. (abatacept). In: Rockville, MD, USA: US Food and Drug Administration; 2005.

2. Weinblatt M, Combe B, Covucci A, Aranda R, Becker JC, Keystone E. Safety of the selective costimulation modulator abatacept in rheumatoid arthritis patients receiving background biologic and nonbiologic disease-modifying antirheumatic drugs: A one-year randomized, placebo-controlled study. Arthritis Rheum 2006;54:2807-16

3. Miller KL, Sawitzke AD, Doane J. Abatacept and serious respiratory infections in patients with previous lung disease. Clin Rheumatol 2008;27:1569-71.

4. Hellings PW, Vandenberghe P, Kasran A, Coorevits L, Overbergh L, Mathieu C, et al. Blockade of CTLA-4 enhances allergic sensitization and eosinophilic airway inflammation in genetically predisposed mice. Eur J Immunol 2002;32:585-94.

5. Deurloo DT, van Esch BC, Hofstra CL, Nijkamp FP, van Oosterhout AJ. CTLA4-IgG reverses asthma manifestations in a mild but not in a more "severe" ongoing murine model. Am J Respir Cell Mol Biol 2001;25:751-60.

6. Lordan JL, Davies DE, Wilson SJ, Dent G, Corkhill A, Jaffar Z, et al. The role of CD28-B7 costimulation in allergen-induced cytokine release by bronchial mucosa from patients with moderately severe asthma. J Allergy Clin Immunol 2001;108:976-81.

J Rheumatol 2012;39:9; doi:10.3899/jrheum.120327 